\title{
CHROMATO-MASS-SPECTROMETRIC RESEARCH IN SALVIA GRANDIFLORA L., SALVIA PRATENSIS L. AND SALVIA VERTICILLATA L. ABOVEGROUND ORGANS
}

\author{
Mykhailo Myha, Oleh Koshovyi, Eugene Karpun, Alla Kovalyova, Olga Mala, \\ Volodymyr Parchenko, Olexandr Panasenko, Vera Bunyatyan, Sergiy Kovalenko
}

\begin{abstract}
The genus Salvia L. has more than 900 species distributed throughout the globe. 21 species are growing in Ukraine. All species of this genus have essential oils. Salvia officinalis and Salvia sclarea have been used in the culture and are widely used in medical practice. The chemical composition of other species of sage and the possibility of their use in pharmaceutical and medical practice are almost not studied. Taking into account the results of chemotaxonomic studies of species of the flora genus of Ukraine, their prevalence and prospects for introduction into the culture, for further studies were selected raw materials of $S$. grandiflora, $S$. pratensis and $S$. verticillata.
\end{abstract}

The aim. The aim of the study was to conduct a chromato-mass spectrometric study of the aboveground organs of S. grandiflora L., S. pratensis L. and S. verticillata L. to establish the prospects for the use of raw materials of these species in medical and pharmaceutical practice.

Materials and methods. The objects of the study were leaves of S. officinalis, leaves, stems and flowers of S. grandiflora, S. pratensis and S. verticillata, which were harvested on the basis of the botanical garden of Ivan Franko National University of Lviv. The research of volatile substances in the objects of the research was carried out by the method of GC-MS on the basis of the Department of Natural Sciences for Foreign Students and Toxicological Chemistry of Zaporizhia State Medical University.

Results. As a result of the study, 243 substances were found in the objects of the study, of which 149 were identified. 77 substances were found in the leaves of S. officinalis, 80, 26 and 63 substances in the leaves, stems and flowers of S. grandiflora, respectively, in the leaves, stems and flowers of S. pratensis - 28, 30 and 48 substances, respectively, in leaves, stems and flowers of S. verticillata - 39, 22 and 39 substances, respectively. Dominant compounds among substances of terpenoid nature are: cyclofenchene, camphene, 1,8-cineole, $\alpha$-thujone, $\beta$-thujone, camphor borneol, caryophyllene, humulene, viridiflorol, sabinene, pyranone, $\beta$-pinene, phytol, kolavenol, $\beta$-copaen, loliolide, pseudolimonene and spatulenol. Among the dominant substances, 8 were detected for the first time in these species: cyclofenchene, viridiflorol, sabinene, pyranone, phytol, kolavenol, loliolide and pseudolimonene.

Conclusions. The leaves of S. officinalis, leaves, stems and flowers of S. grandiflora, S. pratensis and S. verticillata of the flora of Ukraine were studied by chromato-mass spectrometric method. As a result of the study, 243 substances were identified, of which 149 were identified. Promising raw materials containing terpene compounds for $S$. grandiflora there are leaves, and for S. pratensis and S. verticillata - flowers, so they are promising agents for introduction into pharmaceutical practice

Keywords: Salvia, leaves, flowers, stems, terpenes, chromato-mass spectrometry

How to cite:

Myha, M., Koshovyi, O., Karpun, E., Kovalyova, A., Mala, O., Parchenko, V., Panasenko, O., Bunyatyan, V., Kovalenko, S. (2021). Chromato-massspectrometric research in Salvia grandiflora L., Salvia pratensis L. and Salvia verticillata L. aboveground organs. ScienceRise: Pharmaceutical Science, 5 (33), 32-40. doi: http://doi.org/10.15587/2519-4852.2021.242761

(C) The Author(s) 2021

This is an open access article under the Creative Commons CC BY license hydrate

\section{Introduction}

The genus Salvia L. has more than 900 species distributed throughout the globe, of which 21 species are represented in the Ukrainian flora. All species of this genus have essential oils. Sage and sage entered the culture $[1,2]$. The chemical composition of other species of sage and the possibility of their use in pharmaceutical and medical practice are almost not studied.

Biologically active substances of the genus Salvia L. are characterized by a wide range of pharmaco- logical activity, in particular: antioxidant activity [3, 4], improvement of memory and cognitive functions $[5,6]$, hypoglycemic and insulin-like activity [6-8], ability to inhibit angiogenesis and proliferation [9, 10], lowering cholesterol $[10,11]$, reducing symptoms during menopause and menopause [12, 13], antibacterial [14, 15], antidiarrheal [16, 17] and antispasmodic activity [18-20]. This determines the relevance of the study of the genus Salvia for the development of new drugs based on them. 
Based on the literature data and the results of our own research, a chemotaxonomic study of 17 species of the genus Sage (Salvia L.): S. officinalis, S. sclarea, S. grandiflora, S. scabiosifolia, S. glutinosa, S. aethiopis, S. pratensis, S. stepposa, S. Sibthorpii, S. illuminata, S. nemorosa, $S$. pendula, $S$. sylvestris, $S$. nutans, $S$. austriaca, S. verticillata and $S$. cernua to identify promising species and establish the possibility of creating new drugs based on them [21]. In the list of these species, 60 compounds of phenolic nature and 185 compounds of terpenoid nature were found $[21,22]$. The chemical profile of the genus is characterized by phenolic compounds: caffeic and rosmarinic acids [3], flavonoids: cynaroside, kosmosiin, hispidulin and cyrsimaritin [23-25] and terpenoids: $\alpha$ - and $\beta$-pinene, derivatives of camphor (camphene, camphor and borneol) [26], p-cymene, 1,8-cineole and limonene [27, 28]. In addition, the amino acid and monosaccharide composition of sage leaves was studied [29]. Taking into account the results of chemotaxonomic studies and the prospects of their introduction into the culture, the raw materials of S. grandiflora, S. pratensis and $S$. verticillata were selected for further studies.

The aim of the research was to conduct a chromato-mass spectrometric study of the aboveground organs of S. grandiflora L., S. pratensis L. and S. verticillata $L$. to establish the prospects for the use of raw materials of these species in medical and pharmaceutical practice.

\section{Planning (methodology) of the research}

Taking into account the results of chemotaxonomic studies of sage flora of Ukraine [21], which showed the prospects of using S. grandiflora, S. pratensis and S. verticillata in medical and pharmaceutical practice, it is planned to conduct chromato-mass spectrometric study of aboveground organs of these species, to identify promising species raw materials and the possibility of its further use in pharmacy (Fig. 1).

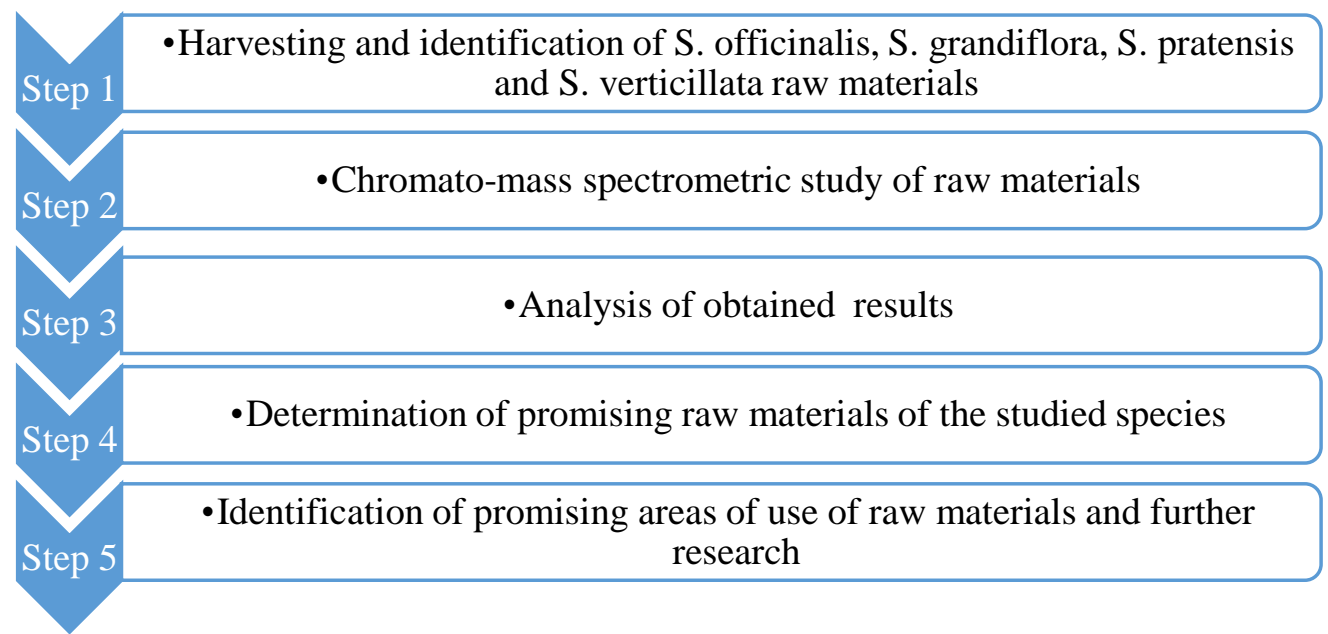

Fig. 1. Scheme of research of S. grandiflora, S. pratensis and S. verticillata raw materials

\section{Materials and methods}

The objects of the study were leaves of S. officinalis, leaves, stems and flowers of S. grandiflora, S. pratensis and S. verticillata, which were harvested on the basis of the Botanical Garden of Ivan Franko National University of Lviv (Lviv, 44 Cheremshyny Street, 79014), under the guidance of a senior researcher, $\mathrm{PhD}$ Skibitska Maria Ivanovna. The studied examples of Salvia genus were identified by PhD Olga Mala from the National University of Pharmacy using a special botanical catalogue [30]. The identified samples corresponded to the moisture content of $\mathrm{SPhU}[31,32]$. The voucher specimens are stored at the Department of Pharmacognosy, The National University of Pharmacy, Kharkiv, Ukraine (No. 1015-1029).

Studies of volatile substances in the objects of the research were carried out by GC-MS method [33] on the basis of the Department of Natural Sciences for Foreign Students and Toxicological Chemistry of Zaporizhia State Medical University according to the method: a sample of 2.0 (exact sample) of plant material was placed in a test tube (volume of which $20 \mathrm{ml}$ ), poured $10 \mathrm{ml}$ of methanol (Sigma-Aldrich) and left for one day. The extract was filtered using a syringe filter, the resulting filtrate was chromatographed $[34,35]$.
Agilent 7890B gas chromatograph with 5977B mass spectrometric detector and Gerstel CIS 4 chilled injection system. DB-5ms chromatographic column $30 \mathrm{~m} \times 250 \mu \mathrm{m} \times 0.25 \mu \mathrm{m}$ long. The speed of the carrier gas (helium) is $1.3 \mathrm{ml} / \mathrm{min}$. The injection volume is $0.5 \mu$ l. Pulse injection with flow division in the ratio 1:5 was used. The temperature of the sampling unit is $200^{\circ} \mathrm{C} \rightarrow 12^{\circ} \mathrm{C} / \mathrm{s} \rightarrow 265^{\circ} \mathrm{C}$. Thermostat temperature: programmable, $70^{\circ} \mathrm{C}(1 \mathrm{~min}$ delay $) \rightarrow 10^{\circ} \mathrm{C} / \mathrm{min} \rightarrow$ $270^{\circ} \mathrm{C}$ (4 min delay). The total chromatography time is 25 minutes. GC/MS interface temperature $-275^{\circ} \mathrm{C}$; ion sources $-230^{\circ} \mathrm{C}$; quadrupole mass analyzer $-150^{\circ} \mathrm{C}$. Type of ionization: electron impact at an electron energy of $70 \mathrm{eV}$. Scanned mass range: $30-700 \mathrm{~m} / \mathrm{z}$. The NIST14 mass spectrum library was used to identify the components. Quantitative determination of the content of substances in the raw material was performed in comparison with a standard sample of menthol $[36,37]$.

\section{Results of the research}

Determination of the qualitative composition and quantitative content of volatile substances in the study objects was performed by GC-MS method using an Agilent 7890B chromatograph. The results of the study of volatile substances in the aboveground organs 
of the studied species of the genus Salvia are given in Table 1. In Fig. 2-5 are shown typical chromatograms obtained in the determination of volatile compounds of the objects of the study.

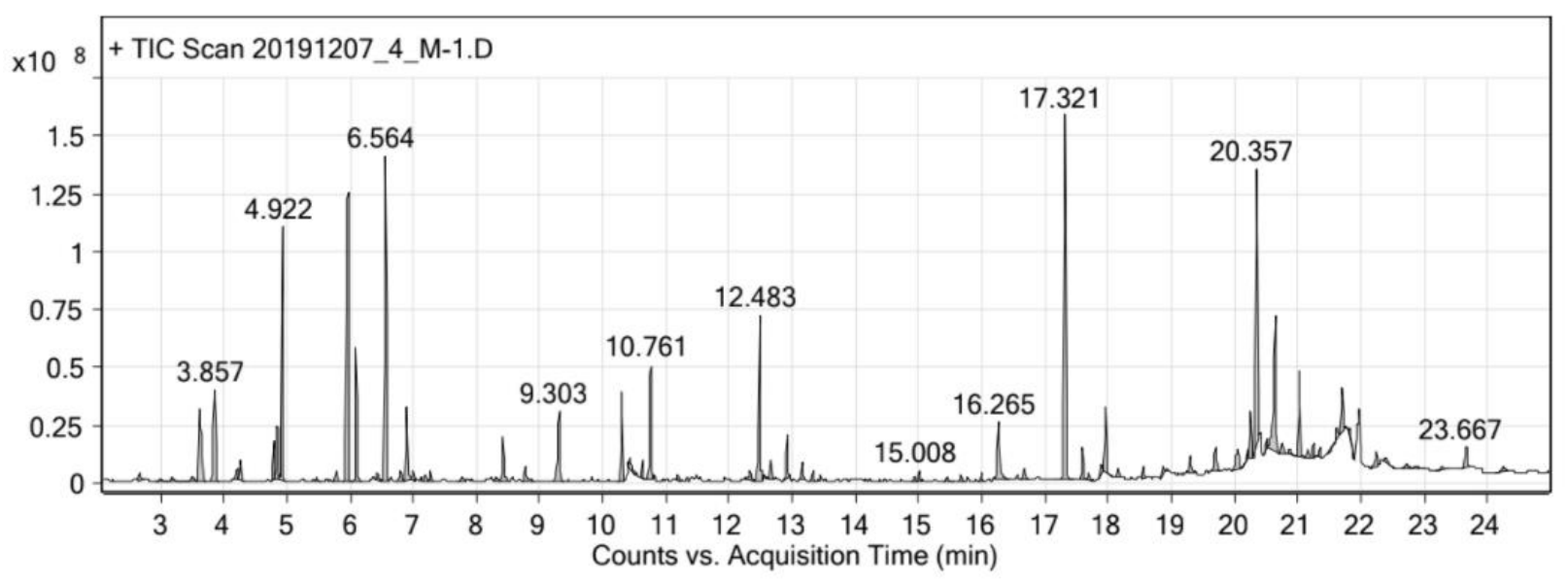

Fig. 2. Typical chromatogram of volatile compounds of S. officinalis leaves

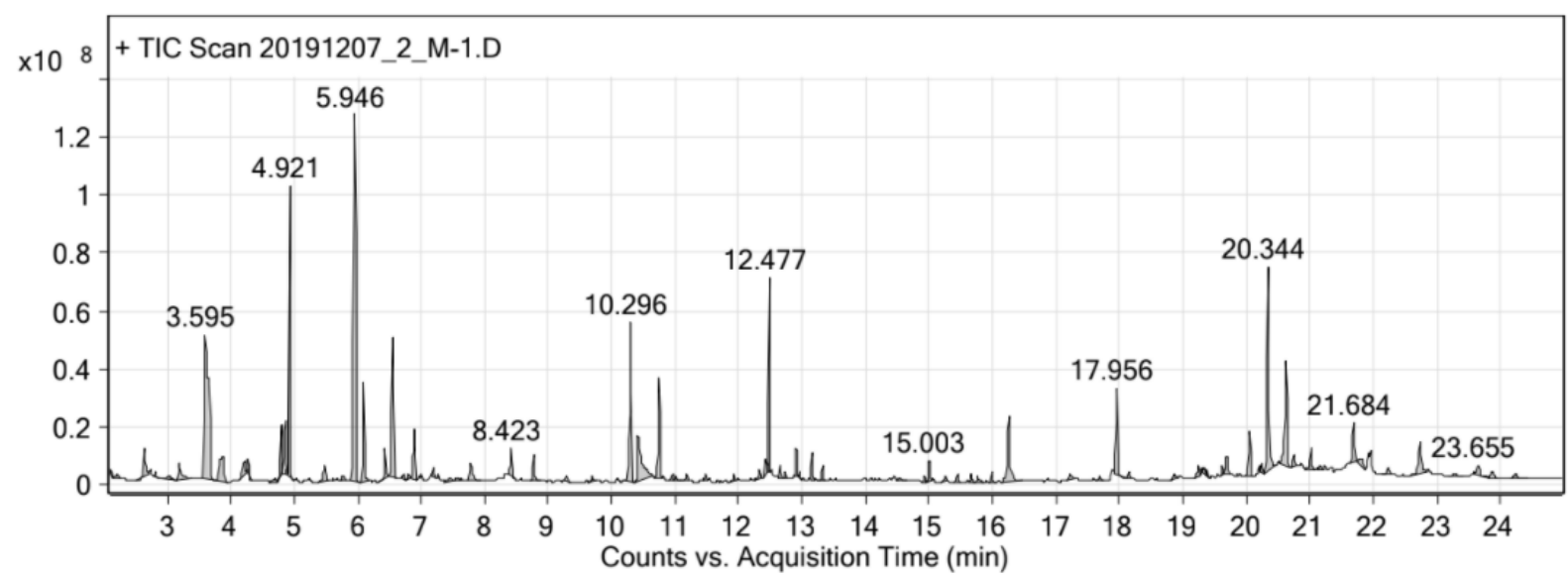

Fig. 3. Typical chromatogram of volatile compounds of S. grandiflora leaves

Table 1

Content of volatile compounds in S. officinalis leaves; leaves, stems and flowers of S. grandiflora, S. pratensis and $\mathrm{S}$. verticillata

\begin{tabular}{|c|c|c|c|c|c|c|c|c|c|c|c|}
\hline & \multirow{3}{*}{ Name of compound } & \multicolumn{10}{|c|}{ Quantitative content, $\mathrm{mg} / 100 \mathrm{~g}$} \\
\hline & & \multirow{2}{*}{$\begin{array}{c}\begin{array}{c}\text { Salvia } \\
\text { officinalis }\end{array} \\
\text { Leaves }\end{array}$} & \multicolumn{3}{|c|}{ Salvia grandiflora } & \multicolumn{3}{|c|}{ Salvia pratensis } & \multicolumn{3}{|c|}{ Salvia verticillata } \\
\hline & & & Leaves & Stem & $\begin{array}{l}\text { Flow- } \\
\text { ers }\end{array}$ & Leaves & Stem & $\begin{array}{l}\text { Flow- } \\
\text { ers }\end{array}$ & $\begin{array}{l}\text { Leav } \\
\text { es }\end{array}$ & Stem & $\begin{array}{l}\text { Flow } \\
\text { ers }\end{array}$ \\
\hline 1 & 2 & 3 & 4 & 5 & 6 & 7 & 8 & 9 & 10 & 11 & 12 \\
\hline 1 & Diepoxybutane & 0 & 1.47 & 0 & 0 & 0 & 0 & 0 & 0 & 0 & 0 \\
\hline 2 & Hydroxyacetone- & 0 & 0 & 0 & 0 & 0 & 1.16 & 0.96 & 0 & 0 & 0 \\
\hline 3 & Methyl acetate & 0 & 0 & 0 & 1 & 0 & 0 & 0 & 0 & 1.06 & 0 \\
\hline 4 & Ethylene glycol, monoacetate & 0 & 0 & 0 & 0 & 1.69 & 0 & 0 & 0 & 0 & 0 \\
\hline 5 & Glycerin & 0 & 2.24 & 0 & 0 & 3.47 & 0 & 3.13 & 0 & 0 & 0 \\
\hline 6 & Glyceraldehyde & 0 & 0 & 2.34 & 6.48 & 0 & 4.56 & 0 & 0 & 10.59 & 2.43 \\
\hline 7 & Cyclopropane, 1,1-dimethyl-2- & 0 & 9.23 & 0 & 0 & 0 & 0 & 0 & 0 & 0 & 0 \\
\hline 8 & $\begin{array}{l}\text { Cyclohexane, } \\
\text { 1,3-dimethyl-2-methylene-, cis- }\end{array}$ & 3.78 & 0 & 0 & 1.29 & 0 & 0 & 0 & 0 & 0 & 0 \\
\hline 9 & Methyl 3-nitropropionate & 2.05 & 3.79 & 2.24 & 2.98 & 0 & 2.55 & 2.77 & 0 & 2.76 & 1.90 \\
\hline 10 & Dihydroxyacetone & 4.54 & 12.01 & 6.23 & 12.41 & 0 & 8.24 & 4.30 & 0 & 12.00 & 4.46 \\
\hline 11 & Tricyclene & 3.99 & 0 & 0 & 0 & 0 & 0 & 0 & 0 & 0 & 0 \\
\hline 12 & $\alpha$-pinene & 0 & 0 & 0 & 0 & 0 & 0 & 0 & 1.84 & 0 & 0 \\
\hline 13 & Cyclofenchene & 61.72 & 140.26 & 6.37 & 38.38 & 0 & 0 & 0 & 0 & 0 & 1.88 \\
\hline 14 & Camphene & 79.72 & 25.77 & 0 & 3.21 & 0 & 0 & 0 & 0 & 0 & 0 \\
\hline
\end{tabular}


Continuation Table 1

\begin{tabular}{|c|c|c|c|c|c|c|c|c|c|c|c|}
\hline \\
\hline 1 & 2 & 3 & 4 & 5 & 6 & 7 & 8 & 9 & 10 & 11 & 12 \\
\hline 15 & $\begin{array}{l}\text { 2,4-dihydroxy-2,5-dimethyl-3(2H)- } \\
\text { furan-3-one }\end{array}$ & 0 & 0 & 0 & 0 & 1.46 & 0 & 2.12 & 0 & 0 & 1.52 \\
\hline 16 & Sabinene & 6.37 & 0 & 6.78 & 0 & 0 & 0 & 0 & 0 & 0 & 0 \\
\hline 17 & Nopinen ( $\beta$-pinene) & 0 & 8.75 & 0 & 10.98 & 0 & 0 & 0 & 0 & 0 & 0 \\
\hline 18 & $\beta$-myrcene & 7.31 & 3.94 & 0 & 1.17 & 0 & 0 & 0 & 0 & 0 & 0 \\
\hline 19 & 4-carene & 0 & 1.97 & 0 & 0 & 0 & 0 & 0 & 0 & 0 & 0.94 \\
\hline 20 & O-cymene & 14.68 & 0 & 0 & 3.68 & 0 & 0 & 0 & 0 & 0 & 0 \\
\hline 21 & M-cymene & 0 & 16.86 & 0 & 0 & 0 & 0 & 0 & 0 & 0 & 0 \\
\hline 22 & D-limonene & 26.78 & 21.88 & 0 & 4.52 & 0 & 0 & 0 & 0 & 0 & 0 \\
\hline 23 & Pseudolimonene & 0 & 0 & 0 & 0 & 0 & 0 & 0 & 0 & 0 & 2.24 \\
\hline 24 & 1,8 -cineole & 116.64 & 104.05 & 6.45 & 35.67 & 0 & 0 & 0 & 0 & 0 & 0 \\
\hline 25 & Levulinic acid & 0 & 0 & 0 & 0 & 0 & 0 & 1.49 & 0 & 0 & 0 \\
\hline 26 & Thymine & 0 & 0 & 0 & 2.32 & 0 & 0 & 0 & 0 & 0 & 0 \\
\hline 27 & D-Alanine & 2.04 & 7.31 & 0 & 0 & 0 & 0 & 4.01 & 0 & 0.92 & 1.47 \\
\hline 28 & Undecan & 0 & 0 & 0 & 0 & 0 & 0 & 0 & 0.78 & 0 & 0 \\
\hline 28 & Linalool & 5.42 & 3.21 & 0 & 0 & 0 & 0 & 0 & 0 & 0 & 0 \\
\hline 30 & $\alpha$-thujone & 171.44 & 186.36 & 36.39 & 40.90 & 0 & 0 & 0 & 1.69 & 0 & 0 \\
\hline 31 & $\beta$-thujone & 57.93 & 42.26 & 6.09 & 9.67 & 0 & 0 & 0 & 0 & 0 & 0 \\
\hline 32 & Pyranone & 3.04 & 9.85 & 6.84 & 5.20 & 7.12 & 15.54 & 14.41 & 0 & 0 & 6.91 \\
\hline 33 & Erythritol & 0 & 0 & 0 & 0 & 0 & 0 & 0 & 0 & 17.38 & 0 \\
\hline 34 & Camphor & 189.82 & 76.31 & 2.68 & 0 & 0 & 0 & 0 & 0.61 & 0 & 0 \\
\hline 35 & 3-pinanone & 0 & 2.01 & 0 & 0 & 0 & 0 & 0 & 0 & 0 & 0 \\
\hline 36 & 5-Caranol & 5.66 & 0 & 0 & 0 & 0 & 0 & 0 & 0 & 0 & 0 \\
\hline 38 & Borneol & 40.24 & 23.83 & 0 & 1.79 & 0 & 0 & 0 & 0 & 0 & 0 \\
\hline 39 & Terpinen-4-ol & 3.58 & 0.00 & 0 & 0.89 & 0 & 0 & 0 & 0 & 0 & 0 \\
\hline 40 & $\alpha$-terpineol & 3.04 & 1.96 & 0 & 1.12 & 0 & 0 & 0 & 0 & 0 & 0 \\
\hline 41 & 1,2,2,3-tetramethylcyclopent-3-enol & 5.06 & 0 & 0 & 0 & 0 & 0 & 0 & 0 & 0 & 0 \\
\hline 42 & Benzofuran & 0 & 0 & 0 & 1.87 & 2.92 & 0 & 5.75 & 0.67 & 0 & 1.07 \\
\hline 43 & Glycerol monoacetate & 2.92 & 11.78 & 4.80 & 8.44 & 7.58 & 9.67 & 15.32 & 5.12 & 7.00 & 5.12 \\
\hline 44 & Phenylacetic acid & 0 & 0 & 0 & 0 & 0 & 0 & 0 & 0.86 & 0 & 0 \\
\hline 45 & 2-butoxyethanol & 0 & 0 & 0 & 0 & 0 & 1.93 & 3.30 & 0 & 1.17 & 2.55 \\
\hline 46 & 6-oxoheptanoic acid & 0 & 0 & 0 & 0 & 2.87 & 0 & 0 & 0 & 1.84 & 0 \\
\hline 47 & 3-hydroxy-2,3-dihydromaltol & 0 & 0 & 0 & 3.25 & 0 & 1.59 & 7.23 & 0 & 0 & 2.69 \\
\hline 48 & Bornylacetate & 23.67 & 11.28 & 0 & 0 & 0 & 0 & 0 & 0 & 0 & 0 \\
\hline 49 & Acetophenone, 2-hydroxy-5-methyl & 9.36 & 10.61 & 0 & 0 & 6.13 & 0 & 5.28 & 1.00 & 0 & 1.25 \\
\hline 50 & 4-hydroxy-3-methylacetophenone & 0 & 0 & 0 & 1.87 & 0 & 1.06 & 0 & 0 & 0 & 0 \\
\hline 51 & Cytramalic acid & 0 & 0 & 0 & 0.93 & 0 & 0 & 0 & 0 & 0 & 0 \\
\hline 52 & $\begin{array}{l}\text { 6-vinyl-3,3-dimethyl-6-hydroxy-5- } \\
\text { formylmethyl-bicyclo [3.2.0]-heptan-2one }\end{array}$ & 38.02 & 1.77 & 0 & 0 & 0 & 0 & 0 & 0 & 0 & 0 \\
\hline 53 & Chavibetol & 0 & 0 & 0 & 0.93 & 0 & 0 & 0 & 0 & 0 & 0 \\
\hline 54 & $\alpha$-copaen & 0 & 2.04 & 0 & 0 & 0 & 0 & 0 & 0 & 0 & 0 \\
\hline 55 & Caryophyllene & 43.43 & 65.96 & 2.39 & 13.79 & 0 & 0 & 12.09 & 14.63 & 0 & 25.51 \\
\hline 56 & Salicylaldehyde hydrazone & 0 & 0 & 0 & 0 & 0 & 0 & 0 & 8.07 & 0 & 0 \\
\hline 57 & 6-methyl salicyaldehyde & 0 & 0 & 0 & 0 & 13.27 & 0 & 0 & 0 & 0 & 0 \\
\hline 58 & Acet fluoroglucin & 7.01 & 0 & 0 & 0 & 0 & 0 & 0 & 0 & 0 & 0 \\
\hline 59 & $\begin{array}{l}\text { 1,5,9,9-tetramethyl-1,4,7, cycloundeca- } \\
\text { triene }\end{array}$ & 0 & 42.61 & 0 & 4.33 & 0 & 0 & 0 & 6.29 & 0 & 11.40 \\
\hline 60 & Humulene & 62.58 & 0 & 0 & 0 & 0 & 0 & 0 & 0 & 0 & 0 \\
\hline 61 & $\gamma$-Murolene & 0 & 2.31 & 0 & 0 & 0 & 0 & 0 & 0 & 0 & 0 \\
\hline 62 & $\begin{array}{l}\text { Ethanone,1-(3-hydroxy-4- } \\
\text { methoxyphenyl) }\end{array}$ & 0 & 1.58 & 0 & 0 & 0 & 0 & 0 & 0 & 0 & 0 \\
\hline 63 & $(-)-\beta$-copaen & 0 & 0 & 0 & 0 & 0 & 0 & 0 & 33.42 & 0 & 0 \\
\hline 64 & Methyl arachidonate & 0 & 2.90 & 0 & 0 & 0 & 0 & 0 & 0 & 0 & 0 \\
\hline 65 & Ledene & 2.79 & 0 & 0 & 0 & 0 & 0 & 0 & 0 & 0 & 0 \\
\hline 67 & 9,10-dihydroxystearate & 0 & 0 & 0 & 0 & 0 & 0 & 0.87 & 0 & 0 & 0 \\
\hline 68 & $\Delta$-cardinene & 0 & 1.58 & 0 & 0 & 0 & 0 & 0 & 0 & 0 & 0 \\
\hline 70 & Dihydroactinolide & 0 & 0 & 0 & 0 & 0 & 0 & 0 & 1.54 & 0 & 0 \\
\hline 71 & Acetoveratron & 0 & 2.06 & 0 & 0 & 0 & 0 & 0 & 0 & 0 & 0 \\
\hline 72 & Spatulenol & 0 & 0 & 0 & 0 & 0 & 0 & 0 & 1.65 & 0 & 2.61 \\
\hline 73 & $\begin{array}{l}\text { 3,5-dihydroxy-6-(hydroxymethyl)oxan- } \\
\text { 2-one }\end{array}$ & 0 & 0 & 0 & 0 & 0 & 2.02 & 0 & 0 & 0 & 0 \\
\hline 74 & Caryophyllene oxide & 4.57 & 3.17 & 0 & 0 & 0 & 0 & 0 & 1.59 & 0 & 1.64 \\
\hline 75 & 1-heptatriacotanol & 0 & 0 & 0 & 0 & 0 & 0 & 1.33 & 0 & 0 & 0 \\
\hline 76 & 3-hydroxy-5,6-epoxy- $\beta$-ionone & 0 & 0 & 0 & 0 & 0 & 0 & 0 & 0.60 & 0 & 0 \\
\hline 77 & Globulol/Ledol/Viridiflorol & 87.35 & 4.44 & 7.57 & 23.47 & 0 & 0 & 0 & 0 & 0 & 0 \\
\hline
\end{tabular}


Continuation Table 1

\begin{tabular}{|c|c|c|c|c|c|c|c|c|c|c|c|}
\hline 1 & 2 & 3 & 4 & 5 & 6 & 7 & 8 & 9 & 10 & 11 & 12 \\
\hline 78 & $\alpha$-gurjunene & 0 & 60.80 & 0 & 0 & 0 & 0 & 0 & 0 & 0 & 0 \\
\hline 79 & Humulene & 2.34 & 0 & 0 & 0 & 0 & 0 & 0 & 0 & 0 & 0 \\
\hline 80 & $\begin{array}{l}\text { (1R,3E,7E,11R)-1,5,5,8-tetramethyl-12- } \\
\text { oxabicyclo [9.1.0]dodeca-3,7-diene }\end{array}$ & 9.13 & 4.06 & 0 & 0 & 0 & 0 & 0 & 0 & 0 & 0 \\
\hline 81 & Tabanone & 0 & 1.75 & 0 & 0 & 0 & 0 & 0 & 0 & 0 & 0 \\
\hline 82 & Diepicedren-1-oxide & 17.35 & 9.67 & 0 & 1.44 & 0 & 0 & 0 & 0 & 0 & 0 \\
\hline 83 & $\begin{array}{l}\text { 11,11-dimethyl-4,8-dimethylenebicyclo- } \\
\text { [7.2.0]undecan-3-ol }\end{array}$ & 0 & 1.43 & 0 & 0 & 0 & 0 & 0 & 0 & 0 & 0 \\
\hline 84 & trans-longipinocarveol & 4.34 & 7.99 & 0 & 0 & 0 & 0 & 0 & 0 & 0 & 0 \\
\hline 85 & Isoaromadendrene epoxide & 6.22 & 0 & 0 & 1.70 & 0 & 0 & 0 & 0 & 0 & 0 \\
\hline 88 & $\begin{array}{l}\text { (1R,7S,E)-7-isopropyl-4,10- } \\
\text { dimethylenecyclodec-5-enol }\end{array}$ & 0 & 5.06 & 0 & 0 & 0 & 0 & 0 & 2.91 & 0 & 0 \\
\hline 89 & Loliolide & 0 & 0 & 0 & 0 & 0 & 0 & 0 & 2.66 & 0 & 0 \\
\hline 90 & Phytol acetate & 3.76 & 11.28 & 0 & 2.28 & 6.04 & 1.69 & 1.54 & 6.63 & 0 & 0 \\
\hline 91 & Phytone & 0 & 0 & 0 & 0 & 0 & 0 & 0 & 0.68 & 0 & 0 \\
\hline 92 & Phthalic acid & 0 & 0 & 0 & 0 & 0 & 0 & 0 & 1.99 & 0 & 0 \\
\hline 93 & $\begin{array}{l}\text { 4,4,8-trimethyltricyclo[6.3.1.0(1,5)] } \\
\text { dodecane-2,9-diol }\end{array}$ & 3.34 & 2.30 & 0 & 0 & 0 & 0 & 0 & 0 & 0 & 0 \\
\hline 94 & Hexadecanoic acid methyl ester & 0 & 0 & 0 & 0 & 0 & 0 & 0 & 0.70 & 2.12 & 0 \\
\hline 95 & Biformen & 3.59 & 3.33 & 0 & 0 & 0 & 0 & 0 & 0 & 0 & 0 \\
\hline 96 & Palmitoleic acid & 35.04 & 37.86 & 10.30 & 13.30 & 62.67 & 22.65 & 40.73 & 41.51 & 7.58 & 21.14 \\
\hline 97 & trans-2-palmitoleic acid & 5.34 & 0 & 0 & 0 & 0 & 0 & 3.13 & 0 & 0 & 0 \\
\hline 98 & 13-epimanool & 169.76 & 0 & 0 & 0 & 0 & 0 & 0 & 0 & 0 & 0 \\
\hline 99 & 8,11-octadecadienoic acid methyl ester & 0 & 0 & 0 & 0 & 0 & 0 & 0 & 0 & 1.14 & 0 \\
\hline 100 & 6,9,12-octadecatrienic acid methyl ester & 0 & 0 & 0 & 0 & 0 & 0 & 0 & 0 & 1.18 & 0 \\
\hline 101 & Glyceryl linoleate & 0 & 0 & 0 & 0 & 0 & 0 & 0 & 0.83 & 0 & 0 \\
\hline 102 & Phytol & 3.05 & 1.91 & 0 & 0 & 8.12 & 0 & 1.51 & 4.75 & 0 & 1.27 \\
\hline 103 & 9,12-octadecadienoic acid (Z,Z) & 3.53 & 0 & 0 & 2.42 & 0 & 1.66 & 3.74 & 0 & 1.59 & 3.40 \\
\hline 104 & 9,12,15-octadecatrienic acid, $(\mathrm{Z}, \mathrm{Z}, \mathrm{Z})$ & 29.38 & 32.34 & 0 & 6.79 & 96.30 & 12.19 & 16.39 & 26.27 & 3.33 & 7.24 \\
\hline 105 & Ascorbyl stearate & 0 & 0 & 0 & 1.17 & 0 & 1.41 & 0 & 0 & 0 & 0 \\
\hline 106 & Stearic acid & 3.99 & 2.35 & 0 & 0 & 4.16 & 0.00 & 6.36 & 3.11 & 0 & 2.00 \\
\hline 107 & Dibutyl sebacate & 0 & 0 & 0 & 0 & 0 & 1.13 & 0 & 0 & 1.04 & 1.11 \\
\hline 108 & Oleic acid & 5.09 & 0 & 4.63 & 0 & 0 & 0 & 0 & 0 & 0 & 0 \\
\hline 109 & Kolavenol & 7.84 & 1.55 & 0 & 0 & 0 & 0 & 2.77 & 0 & 0 & 0 \\
\hline 110 & $\begin{array}{l}\text { 1,4-dimethyl-8- } \\
\text { isopropylidenetricy- } \\
\text { clo[5.3.0.0(4.10)]decane }\end{array}$ & 12.36 & 1.88 & 0 & 0 & 0 & 0 & 0 & 0 & 0 & 0 \\
\hline 111 & Dehydroabietane & 0 & 1.64 & 0 & 0 & 0 & 0 & 0 & 0 & 0 & 0 \\
\hline 112 & $\begin{array}{l}\text { Podocarpa,8,11,13-tetraene-3one,14- } \\
\text { isopropyl-1,13-dimethoxy }\end{array}$ & 13.53 & 8.00 & 0 & 5.27 & 0 & 0 & 0 & 0 & 0 & 0 \\
\hline 113 & 12-O-methylcarnosol & 14.26 & 17.98 & 3.96 & 5.67 & 0 & 0 & 0 & 0 & 0 & 0 \\
\hline 114 & 11-decyldocasane & 0 & 0 & 0 & 0 & 0 & 0 & 1.61 & 0 & 0 & 0 \\
\hline 115 & Isocarnosol & 19.62 & 0 & 0 & 0 & 0 & 0 & 0 & 0 & 0 & 0 \\
\hline 116 & Galantamine & 181.55 & 89.06 & 16.26 & 30.93 & 0 & 0 & 0 & 0 & 0 & 0 \\
\hline 117 & Equiline derivat & 0 & 0 & 0 & 0 & 1.80 & 1.20 & 0 & 0 & 0 & 0 \\
\hline 118 & $\begin{array}{l}\text { 1,2,3,4-tetrahydro-1,4- } \\
\text { ethanoanthracene,9,10-dimethoxy- }\end{array}$ & 3.56 & 0 & 0 & 0 & 0 & 0 & 0 & 0 & 0 & 0 \\
\hline 119 & $\begin{array}{l}\text { 1- (4-methyl [1,1 ': 4', } 1 \text { "] terphenyl- } \\
\text { 4"yl) ethanone }\end{array}$ & 0 & 52.18 & 0 & 0 & 0 & 0 & 0 & 0 & 0 & 0 \\
\hline 120 & Atrovenetrin & 0 & 3.69 & 0 & 0 & 0 & 0 & 0 & 0 & 0 & 0 \\
\hline 121 & Squalene & 0 & 0 & 0 & 2.91 & 0 & 0 & 0 & 0 & 0 & 0 \\
\hline 122 & Octadecane, 3-ethyl-5-(2-ethylbutyl)- & 0 & 0 & 0 & 0 & 0 & 0 & 3.81 & 0.59 & 0 & 0 \\
\hline 126 & 1,2-dipalmitin & 0 & 0 & 0 & 0 & 1.52 & 0 & 0 & 0 & 0 & 0 \\
\hline 128 & Ethyl isocholate & 0 & 0 & 0 & 0 & 1.88 & 0 & 0 & 3.71 & 0 & 0 \\
\hline 129 & $\begin{array}{l}\text { Dibenz[a,c]cyclohexane, } \\
\text { trimethoxy- }\end{array}$ & 4.22 & 0 & 0 & 0 & 0 & 0 & 0 & 0 & 0 & 0 \\
\hline 130 & Pizyferol & 19.53 & 15.38 & 2.70 & 9.70 & 0 & 0 & 0 & 0 & 0 & 0 \\
\hline 131 & $\begin{array}{l}\begin{array}{l}\text { Dibenz[d, f]cycloheptanone, } \\
\text { trimethoxy- }\end{array} \\
\end{array}$ & 7.56 & 0 & 0 & 0 & 0 & 0 & 0 & 0 & 0 & 0 \\
\hline 132 & $\gamma$-sitosterol & 2.20 & 0 & 11.92 & 14.81 & 36.78 & 18.51 & 5.56 & 0 & 13.31 & 18.68 \\
\hline 133 & Germanicol & 0 & 0 & 0 & 0 & 0 & 1.45 & 0 & 0 & 0 & 0 \\
\hline 134 & 12-O-Methylcarnosol & 3.16 & 20.31 & 0 & 2.57 & 0 & 0 & 0 & 0 & 0 & 0 \\
\hline 135 & $\begin{array}{|llr|}\begin{array}{l}\text { Dibenz[a, } \\
\text { trimethoxy- }\end{array} & \text { c]cycloheptane, } & \text { 2,3,7- } \\
\end{array}$ & 2.55 & 0 & 0 & 0 & 0 & 0 & 0 & 0 & 0 & 0 \\
\hline
\end{tabular}


Continuation Table 1

\begin{tabular}{|c|c|c|c|c|c|c|c|c|c|c|c|c|}
\hline 1 & \multicolumn{2}{|c|}{2} & 3 & 4 & 5 & 6 & 7 & 8 & 9 & 10 & 11 & 12 \\
\hline 136 & \multicolumn{2}{|c|}{$\begin{array}{l}\text { 6a, 14a-methanopicene, perhydro-1,2,4a, } \\
\text { 6b, 9,9,12a-hexamethyl-10-hydroxy- }\end{array}$} & 0 & 0 & 0 & 0 & 0 & 20.02 & 0 & 0 & 5.37 & 0 \\
\hline 137 & \multicolumn{2}{|c|}{ Salvigenin } & 0 & 0 & 5.85 & 0 & 0 & 0 & 0 & 0 & 0 & 2.87 \\
\hline 138 & \multicolumn{2}{|l|}{$\alpha$-amyrin } & 0 & 0 & 0 & 0 & 0 & 7.95 & 0 & 0 & 0 & 0 \\
\hline 140 & \multicolumn{2}{|l|}{$\alpha$-tocopherol } & 0 & 0 & 9.57 & 0 & 11.39 & 0 & 0 & 0 & 0 & 0 \\
\hline 141 & \multicolumn{2}{|l|}{ 9-octylhexacosane } & 0 & 0 & 0 & 0 & 0 & 0 & 1.58 & 0 & 0 & 0 \\
\hline 142 & \multicolumn{2}{|l|}{ Supraene } & 4.26 & 3.09 & 0 & 0 & 2.65 & 0 & 0 & 0 & 0 & 1.11 \\
\hline 143 & \multicolumn{2}{|c|}{$\begin{array}{l}\text { 2,6,6,9,2',6',6',9'-octamethyl- } \\
\text { [8,8']bi[tricyclo[5.4.0.0(2,9)]undecyl] }\end{array}$} & 0 & 0 & 0 & 0 & 0 & 9.97 & 0 & 0 & 0 & 0 \\
\hline 144 & \multicolumn{2}{|c|}{ Betulinaldehyde } & 0 & 0 & 0 & 0 & 0 & 7.13 & 0 & 0 & 0 & 0 \\
\hline 148 & \multicolumn{2}{|c|}{ 3-methoxyergost-8(14)-ene } & 0 & 0 & 16.77 & 0 & 0 & 0 & 0 & 0 & 0 & 0 \\
\hline 149 & \multicolumn{2}{|c|}{ Ursolic aldehyde } & 0 & 0 & 0 & 16.45 & 0 & 0 & 0 & 0 & 0 & 0 \\
\hline \multirow{2}{*}{\multicolumn{2}{|c|}{ Unidentified substances }} & pcs. & 15 & 19 & 4 & 21 & 8 & 8 & 21 & 9 & 5 & 12 \\
\hline & & $\mathrm{mg} / 100 \mathrm{~g}$ & 152.04 & 86.08 & 24.86 & 48.09 & 29.17 & 8.73 & 56.39 & 22.34 & 14.29 & 34.79 \\
\hline
\end{tabular}

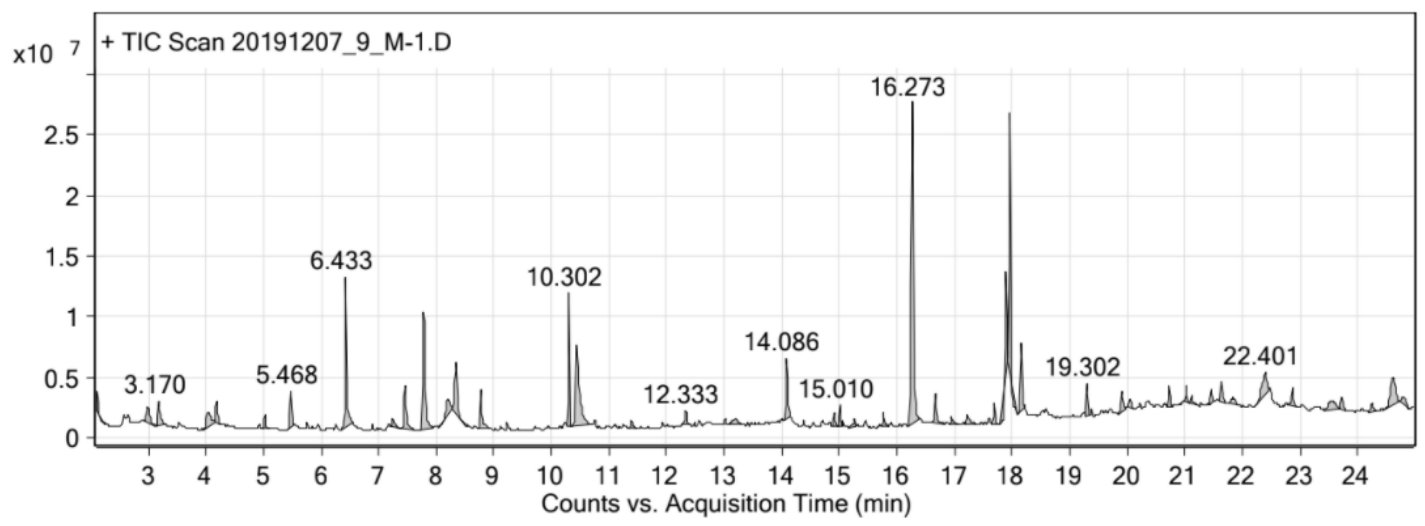

Fig. 4. Typical chromatogram of volatile compounds of S. pratensis flowers

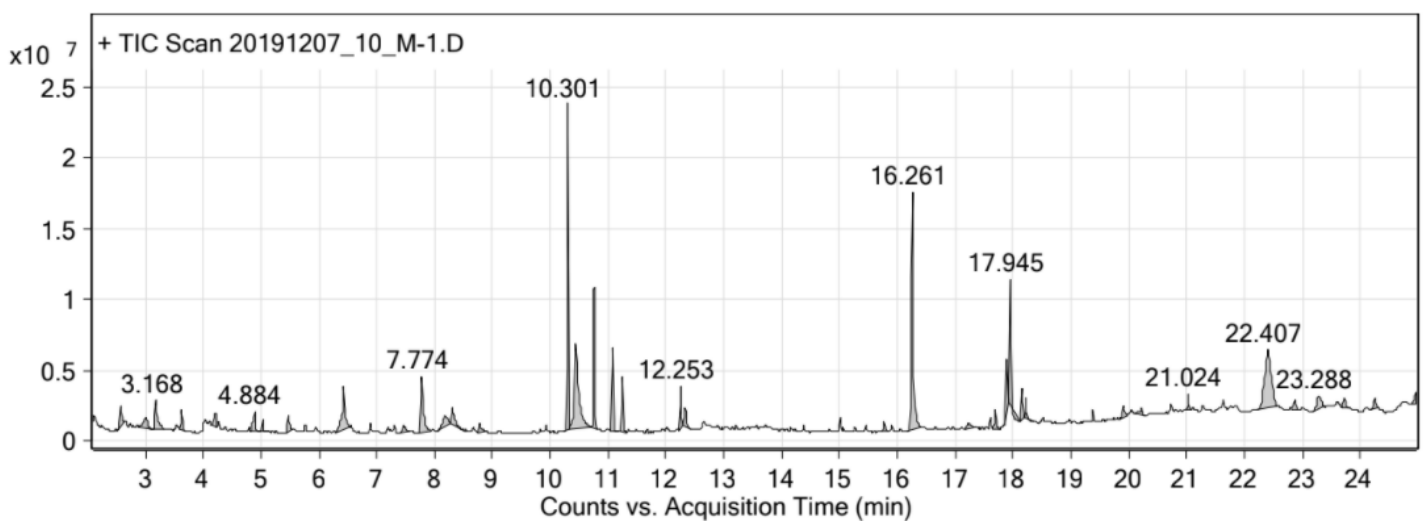

Fig. 5. Typical chromatogram of volatile compounds of S. verticillata flowers

As a result of the study, 243 substances were found in the objects of the study, of which 149 were identified.

\section{Discussion of the results}

77 substances were found in the leaves of S. officinalis, the dominant ones were cyclofenchene, camphene, 1,8 -cineole, $\alpha$-thujone, $\beta$-thujone, camphor borneol, caryophyllene, humulene, viridiflorol and 13-epimanool.

In the leaves of $\mathrm{S}$. grandiflora 80 substances were found, the dominant ones were cyclofenchene, 1,8-cineole, $\alpha$-thujone, $\beta$-thujone, camphor borneol, caryophyllene and viridiflorol. 26 substances were found in the stem of S. grandiflora, the dominant ones were cyclofenchene, sabinene, 1,8-cineole, $\alpha$ - and $\beta$-thujone and pyranone. 63 substances were found in S. grandiflora flowers, the dominant ones being cyclofenchene, $\beta$-pinene, 1,8 -cineole, $\alpha$-thujone, caryophyllene and viridiflorol.

In the leaves of $\mathrm{S}$. pratensis 28 substances were found, pyranone and phytol were dominant. 30 substances were found in the stem of S. pratensis, pyranone and $\alpha$-amyrin were dominant. 48 substances were found in S. pratensis flowers, pyranone, caryophyllene, phytol and kolavenol were dominant.

In the leaves of $\mathrm{S}$. verticillata 39 substances were found, the dominant ones were caryophyllene, $\beta$-copaen, loliolide and phytol. 22 substances were found in the stem of S. verticillata. In S. verticillata flowers 39 substances were found, the dominant ones were cyclofenchene, pseudolimonene, pyranone, caryophyllene, spatulenol and phytol.

Dominant compounds among substances of terpenoid nature are: cyclofenchene, camphene, 1,8-cineole, $\alpha$ - 
thujone, $\beta$-thujone, camphor borneol, caryophyllene, humulene, viridiflorol, sabinene, pyranone, $\beta$-pinene, phytol, kolavenol, $\beta$-pinene, loliolide, pseudolimonene and spatulenol. Among the dominant substances, 8 were detected for the first time in these species: cyclofenchene, viridiflorol, sabinene, pyranone, phytol, kolavenol, loliolide and pseudolimonene.

The raw materials of all three studied species $\mathrm{S}$. grandiflora, S. pratensis and S. verticillata are rich in caryophyllene, so they could be potential sources of it. The widespread plant volatile beta-caryophyllene (BCP) was recently identified as a natural selective agonist of the peripherally expressed cannabinoid receptor 2 (CB2). Number of studies have shown that CB2 is critically involved in the modulation of inflammatory and neuropathic pain responses. Thus, the natural plant product $\mathrm{BCP}$ may be highly effective in the treatment of long lasting, debilitating pain states [38]. More importantly, BCP inhibited EtOH-CPP acquisition and exacerbated LORR duration. Interestingly, these effects were abrogated when mice were pre-injected with a selective CB2 receptor antagonist, AM630. Overall, the CB2 receptor system appears to be involved in alcohol dependence and sensitivity and may represent a potential pharmacological target for the treatment of alcoholism [39]. Moreover, oral administration of $\mathrm{BCP}$ effectively rescued $\beta$-cells by mitigating hyperglycemia through enhancing insulin release and also averted oxidative/inflammatory stress in pancreatic tissue of diabetic rats. The efficacy of BCP was compared with glibenclamide, a standard antidiabetic drug [40]. The characterization tests indicated that $\beta \mathrm{CP}$ were efficiently incorporated into $\beta C D$. The oral treatment with $\beta C P-\beta C D$, at all doses tested, produced a significant $(\mathrm{p}<0.05)$ reduction on mechanical hyperalgesia and a significant $(\mathrm{p}<0.05)$ increase in muscle withdrawal thresholds, without produce any alteration in force. In addition, $\beta C P-\beta C D$ was able to significantly $(\mathrm{p}<0.05)$ decrease Fos expression in the superficial dorsal horn. Thus, $\beta C P-\beta C D$ attenuates the noninflammatory chronic muscle pain in mice and inhibits the Fos expression in the lumbar spinal cord [41].

In addition, all three studied species S. grandiflora, S. pratensis and S. verticillata are rich in phytol and its derivatives, so they could be potential sources of it. In the pharma-medico viewpoint, PYT and its derivatives have been evident to have antimicrobial, cytotoxic, antitumorous, antimutagenic, anti-teratogenic, antibioticchemotherapeutic, antidiabetic, lipid lowering, antispasmodic, anticonvulsant, antinociceptive, antioxi-dant, antiinflammatory, anxiolytic, antidepressant, immunoadjuvancy, hair growth facilitator, hair fall defense and antidandruff activities. Otherwise, the important biometebolite of PYT is phytanic acid (PA). Evidence shows PA to have cytotoxic, anticancer, antidiabetic, lipid lowering and aniteratogenic activities. In addition, it may be considered as an important biomarker for some diseases such as Refsum's Disease (RD), Sjögren Larsson syndrome (SLS), rhizomelic chondrodysplasia punctata (RZCP), chronic polyneuropathy (CP), Zellweger's disease hyperpipecolic academia (ZDHA) and related diseases. Thus, phytol may be considered as a new drug candidate $[42,43]$.

The raw materials of the studied species S. grandiflora, S. pratensis and S. verticillata are rich in $\gamma$-Sitosterol, so they could be potential sources of it. The study demon- strates in vitro results, which support the ethnomedical use of $\gamma$-Sitosterol against cancer. Experimental results of this study suggest that $\gamma$-Sitosterol exerts potential anticancer activity through the growth inhibition, cell cycle arrest and the apoptosis on cancer cells [44]. $\gamma$-sitosterol was screened for its antidiabetic property in streptozotocin (STZ) induced diabetic rats. Furthermore $\gamma$-sitosterol showed antihyperlipidemic activity as evidenced by significant decrease in serum total cholesterol, triglycerides and very low density lipoprotein-cholesterol levels coupled with elevation of high density lipoprotein-cholesterol levels in treated rats. A significant decrease in the activities of alanine aminotransaminase, aspartate aminotransaminase, alkaline phosphatase and acid phosphatase in $\gamma$-sitosterol treated rats when compared to diabetic control rats indicated its protective role against liver damage. $\gamma$-Sitosterol increased insulin secretion in response to glucose. Immunohistochemical study of pancreas also confirmed the biochemical findings. These results indicated that $\gamma$-sitosterol possessed antihyperglycemic activity [45].

Given the qualitative composition and quantitative content of terpene substances, promising raw materials for S. grandiflora are leaves, and for S. pratensis and S. verticillata - flowers, so they are promising agents for introduction into pharmaceutical practice.

Study limitations. Not all substances could be identified during the GC MS study of raw materials, as they were not available in the library that was used.

The prospects for the further research. To create new drugs, it is advisable to conduct a more thorough phytochemical study of raw materials of the studied species of sage, to standardize these raw materials and obtain galenic drugs based on it.

\section{Conclusions}

The leaves of S. officinalis, leaves, stems and flowers of S. grandiflora, S. pratensis and S. verticillata of the flora of Ukraine were studied by chromato-mass spectrometric method. As a result of the study, 243 substances were identified, of which 149 were identified. The dominant compounds among terpenoid substances are: cyclofenchene, camphene, 1,8-cineole, $\alpha$-thujone, $\beta$-thujone, camphor borneol, caryophyllene, humulene, viridiflorol, sabinene, pyranone, $\beta$-pinene, phytol, kolavenol, $\beta$-copaen, loliolide, pseudolimonene and spatulenol. Among the dominant substances, 8 were detected for the first time in these species: cyclofenchene, viridiflorol, sabinene, pyranone, phytol, kolavenol, loliolide and pseudolimonene.

A promising raw material containing terpene compounds for $\mathrm{S}$. grandiflora are leaves, and for $\mathrm{S}$. pratensis and S. verticillata - flowers, so they are promising agents for introduction into pharmaceutical practice.

\section{Conflict of interests.}

Authors declare there is no conflict of interest.

\section{Funding.}

The research was funded by the Ministry of Health Care of Ukraine at the expense of the State Budget in the framework No. 2301020 "Scientific and scientific-technical activity in the field of health protection" on the topic "Modern approaches to the creation of new medicines for a correction of metabolic syndrome". 


\section{References}

1. Komarov, V. L. (1954). Shalfei- Salvia. Flora SSSR. Moscow-Leningrad, 21, 244-374.

2. Komarov, V. L. (1991). Rastitelnye resursy SSSR: TSvetkovye rasteniia, ikh khimicheskii sostav, ispolzovanie. Saint Petersburg: Nauka, 200.

3. Kamatou, G. P. P., Viljoen, A. M., Steenkamp, P. (2010). Antioxidant, antiinflammatory activities and HPLC analysis of South African Salvia species. Food Chemistry, 119 (2), 684-688. doi: http://doi.org/10.1016/j.foodchem.2009.07.010

4. Shanaida, M., Hudz, N., Korzeniowska, K., Wieczorek, P. P. (2018). Antioxidant activity of essential oils obtained from aerial part of some Lamiaceae species. International Journal of Green Pharmacy, 12 (3), 200-204.

5. Eidi, M., Eidi, A., Bahar, M. (2006). Effects of Salvia officinalis L. (sage) leaves on memory retention and its interaction with the cholinergic system in rats. Nutrition, 22 (3), 321-326. doi: http://doi.org/10.1016/j.nut.2005.06.010

6. Tildesley, N., Kennedy, D., Perry, E., Ballard, C., Wesnes, K., Scholey, A. (2005). Positive modulation of mood and cognitive performance following administration of acute doses of Salvia lavandulaefolia essential oil to healthy young volunteers. Physiology \& Behavior, 83 (5), 699-709. doi: http://doi.org/10.1016/j.physbeh.2004.09.010

7. Mahdizadeh, R., Moein, S., Soltani, N., Malekzadeh, K., Moein, M. (2018). Study of molecular mechanism of Salvia species in prevention of diabetes. International Journal of Pharmaceutical Sciences and Research, 9, 4512-4521. doi: http://doi.org/10.13040/ ijpsr.0975-8232.9(11).4512-21

8. Eidi, M., Eidi, A., Zamanizadeh, H. (2005). Effect of Salvia officinalis L. leaves on serum glucose and insulin in healthy and streptozotocin-induced diabetic rats. Journal of Ethnopharmacology, 100 (3), 310-313. doi: http://doi.org/10.1016/j.jep. 2005.03.008

9. Bjarnason, I., Scarpignato, C., Holmgren, E., Olszewski, M., Rainsford, K. D., Lanas, A. (2018). Mechanisms of Damage to the Gastrointestinal Tract From Nonsteroidal Anti-Inflammatory Drugs. Gastroenterology, 154 (3), 500-514. doi: http://doi.org/10.1053/j.gastro.2017.10.049

10. Ghorbani, A., Esmaeilizadeh, M. (2017). Pharmacological properties of Salvia officinalis and its components. Journal of Traditional and Complementary Medicine, 7 (4), 433-440. doi: http://doi.org/10.1016/j.jtcme.2016.12.014

11. Zagayko, A. L., Kolisnyk, T. Y., Chumak, O. I., Ruban, O. A., Koshovyi, O. M. (2018). Evaluation of anti-obesity and lipid-lowering properties of Vaccinium myrtillus leaves powder extract in a hamster model. Journal of Basic and Clinical Physiology and Pharmacology, 29 (6), 697-703. doi: http://doi.org/10.1515/jbcpp-2017-0161

12. Nizhenkovska, I. V., Tsurkan, O. O., Sedko, K. V. (2014). Shavliia likarska - suchasni aspekty zastosuvannia (Ohliad literatury). Fitoterapiia. Chasopys, 2, 58-61.

13. Raja, R. R. (2012). Medicinally Potential Plants of Labiatae (Lamiaceae) Family: An Overview. Research Journal of Medicinal Plant, 6 (3), 203-213. doi: http://doi.org/10.3923/rjmp.2012.203.213

14. Koshovyi, O. N., Vovk, G. V., Akhmedov, E. Yu., Komissarenko, A. N. (2015). The study of the chemical composition and pharmacological activity of Salvia officinalis leaves extracts getting by complex processing. Azerbaijan Pharmaceutical and Pharmacotherapy Journal, 15 (1), 30-34.

15. Verkhovodova, Yu., Kireyev, I., Koshovyi, O., Myha, M., Osolodchenko, T. (2020). The effect of common sage extracts on the intestinal microbiota in experimental infectious colitis. Georgian Medical News, 4 (301), 165-170.

16. Nikavar, B., Abolhasani, L., Izadpanah, H. (2008). Alpha-amylase inhibitory activities of six salvia species. Iranian Journal of Pharmaceutical Research, 7, 297-303. doi: http://doi.org/10.22037/IJPR.2010.779

17. Khan, A., Rehman, N., AlKharfy, K. M., Gilani, A.-H. (2011). Antidiarrheal and antispasmodic activities of Salvia officinalis are mediated through activation of $\mathrm{K}+$ channels. Bangladesh Journal of Pharmacology, 6 (2), 111-116. doi: http://doi.org/10.3329/bjp.v6i2.9156

18. Hamidpour, M., Hamidpour, R., Hamidpour, S., \& Shahlari, M. (2014). Chemistry, Pharmacology, and Medicinal Property of Sage (Salvia) to Prevent and Cure Illnesses such as Obesity, Diabetes, Depression, Dementia, Lupus, Autism, Heart Disease, and Cancer. Journal of Traditional and Complementary Medicine, 4 (2), 82-88. doi: http://doi.org/10.4103/2225-4110.130373

19. Mashkovskii, M. D. (2010). Lekarstvennye sredstva. Moscow: OOO «Izd-vo Novaia Volna», 1216.

20. Kovalenko, V. N. (2020). Compendium 2020 - Medicines. Kyiv: MORION, 2700.

21. Koshovyi, O., Raal, A., Kovaleva, A., Myha, M., Ilina, T., Borodina, N., Komissarenko, A. (2020). The phytochemical and chemotaxonomic study of Salvia spp. growing in Ukraine. Journal of Applied Biology \& Biotechnology, 8 (3), 29-36. doi: http://doi.org/10.7324/jabb.2020.80306

22. Semenchenko, O. M. Tsurkan, O. O., Korablova, O. A., Burmaka, O. V. (2013). Determination of volatile compounds of essential oils of different species of genus of Salvia by chromatography-mass spectrometric method. Farmatsevtychnyi zhurnal, 1, 62-65.

23. Jasicka-Misiak, I., Poliwoda, A., Petecka, M., Buslovych, O., Shlyapnikov, V. A., Wieczorek, P. P. (2018). Antioxidant Phenolic Compounds in Salvia officinalis L. and Salvia sclarea L. Ecological Chemistry and Engineering S, 25 (1), 133-142. doi: http://doi.org/10.1515/eces-2018-0009

24. Kharazian, N. (2013). Identification of flavonoids in leaves of seven wild growing Salvia L. (Lamiaceae) species from ?ran. Progress in Biological Sciences, 3 (2), 81-98. doi: http://doi.org/10.22059/PBS.2013.35842

25. Myha, M., Koshovyi, O., Gamulya, O., Ilina, T., Borodina, N., Vlasova, I. (2020). Phytochemical study of Salvia grandiflora and Salvia officinalis leaves for establishing prospects for use in medical and pharmaceutical practice. ScienceRise: Pharmaceutical Science, 1 (23), 23-28. doi: http://doi.org/10.15587/2519-4852.2020.197299

26. Rami, K., Zheng Guo, L. (2011). Antimicrobial activity of essential oil of Salvia officinalis L. collected in Syria. African Journal of Biotechnology, 10 (42), 8397-8402. doi: http://doi.org/10.5897/ajb10.2615

27. Khedher, M. R., Khedher, S. B., Chaieb, I., Tounsi, S., Hammami, M. (2017). Chemical composition and biological activities of Salvia officinalis essential oil from Tunisia. EXCLI Journal, 16, 160-173. doi: http://doi.org/10.17179/excli2016-832

28. Gericke, S., Lübken, T., Wolf, D., Kaiser, M., Hannig, C., Speer, K. (2018). Identification of New Compounds from Sage Flowers (Salvia officinalis L.) as Markers for Quality Control and the Influence of the Manufacturing Technology on the Chemical Composition and Antibacterial Activity of Sage Flower Extracts. Journal of Agricultural and Food Chemistry, 66 (8), $1843-1853$. doi: http://doi.org/10.1021/acs.jafc.8b00581

29. Koshevoi, O. N. (2011). Amino-acid and monosaccharide compositions of Salvia officinalis leaves. Chemistry of Natural Compounds, 47 (3), 492-493. doi: http://doi.org/10.1007/s10600-011-9976-3

30. Dobrochaeva, D. N., Kotox, M. I., Prokudin, Y. N., Barbarich, A. I. (1999). Opredelitel vysshikh rastenii Ukrainy . Kyiv: Naukova dumka.

31. Koshevoy, N., Zabolotny, A., Koshevaya, I., Kostenko, E. M., Rozhnova, T. (2019). Research of moisture-meter device for bulk and liquid materials. 29th International Scientific Symposium Metrology and Metrology, MMA. doi: http://doi.org/10.1109/ mma.2019.8935983

32. Korobiichuk, I., Bezvesilna, O., Kachniarz, M., Koshovyj, M., Kvasnikov, V. (2018). Methods and Ways of Piezoelectric Accelerometers Fastening on the Objects of Research. Acta Physica Polonica A, 133 (4), 1112-1115. doi: http://doi.org/10.12693/ aphyspola.133.1112

33. Derzhavna Farmakopeia Ukrainy. Vol. 1. (2015). Kharkiv: Derzhavne pidpryiemstvo «Ukrainskyi naukovyi farma?opeinyi tsentr yakosti likarskykh zasobiv», 1128. 
34. Krivoruchko, E., Markin, A., Samoilova, V. A., Ilina, T., Koshovyi, O. (2018). Research in the chemical composition of the bark of sorbus aucuparia. Ceska a Slovenska Farmacie, 67 (3), 113-115.

35. Starchenko, G., Hrytsyk, A., Raal, A., Koshovyi, O. (2020). Phytochemical Profile and Pharmacological Activities of Water and Hydroethanolic Dry Extracts of Calluna vulgaris (L.) Hull. Herb. Plants, 9 (6), 751. doi: http://doi.org/10.3390/plants9060751

36. Shinkovenko, I. L., Kashpur, N. V., Ilyina, T. V., Kovalyova, A. M., Goryacha, O. V. et. al. (2018). The immun?modulatory activity of the extracts and complexes of biologically active compounds of Galium verum L. herb. Ceska a Slovenska Farmacie, 67 (1), 25-29.

37. Koshovyi, O., Raal, A., Kireyev, I., Tryshchuk, N., Ilina, T., Romanenko, Y. et. al. (2021). Phytochemical and Psychotropic Research of Motherwort (Leonurus cardiaca L.) Modified Dry Extracts. Plants, 10 (2), 230. doi: http://doi.org/10.3390/plants10020230

38. Klauke, A.-L., Racz, I., Pradier, B., Markert, A., Zimmer, A. M., Gertsch, J., Zimmer, A. (2014). The cannabinoid CB2 receptor-selective phytocannabinoid beta-caryophyllene exerts analgesic effects in mouse models of inflammatory and neuropathic pain. European Neuropsychopharmacology, 24 (4), 608-620. doi: http://doi.org/10.1016/j.euroneuro.2013.10.008

39. Al Mansouri, S., Ojha, S., Al Maamari, E., Al Ameri, M., Nurulain, S. M., Bahi, A. (2014). The cannabinoid receptor 2 agonist, $\beta$-caryophyllene, reduced voluntary alcohol intake and attenuated ethanol-induced place preference and sensitivity in mice. Pharmacology Biochemistry and Behavior, 124, 260-268. doi: http://doi.org/10.1016/j.pbb.2014.06.025

40. Basha, R. H., Sankaranarayanan, C. (2016). $\beta$-Caryophyllene, a natural sesquiterpene lactone attenuates hyperglycemia mediated oxidative and inflammatory stress in experimental diabetic rats. Chemico-Biological Interactions, 245, 50-58. doi: http://doi.org/10.1016/j.cbi.2015.12.019

41. Quintans-Júnior, L. J., Araújo, A. A. S., Brito, R. G., Santos, P. L., Quintans, J. S. S., Menezes, P. P. et. al. (2016). $\beta$ caryophyllene, a dietary cannabinoid, complexed with $\beta$-cyclodextrin produced anti-hyperalgesic effect involving the inhibition of Fos expression in superficial dorsal horn. Life Sciences, 149, 34-41. doi: http://doi.org/10.1016/j.lfs.2016.02.049

42. Islam, M. T., de Alencar, M. V. O. B., da Conceição Machado, K., da Conceição Machado, K., de Carvalho MeloCavalcante, A. A., de Sousa, D. P., de Freitas, R. M. (2015). Phytol in a pharma-medico-stance. Chemico-Biological Interactions, 240, 60-73. doi: http://doi.org/10.1016/j.cbi.2015.07.010

43. Islam, M. T., Ali, E. S., Uddin, S. J., Shaw, S., Islam, M. A., Ahmed, M. I. et. al. (2018). Phytol: A review of biomedical activities. Food and Chemical Toxicology, 121, 82-94. doi: http://doi.org/10.1016/j.fct.2018.08.032

44. Sundarraj, S., Thangam, R., Sreevani, V., Kaveri, K., Gunasekaran, P., Achiraman, S., Kannan, S. (2012). $\gamma$-Sitosterol from Acacia nilotica L. induces G2/M cell cycle arrest and apoptosis through c-Myc suppression in MCF-7 and A549 cells. Journal of Ethnopharmacology, 141 (3), 803-809. doi: http://doi.org/10.1016/j.jep.2012.03.014

45. Balamurugan, R., Duraipandiyan, V., Ignacimuthu, S. (2011). Antidiabetic activity of $\gamma$-sitosterol isolated from Lippia nodiflora L. in streptozotocin induced diabetic rats. European Journal of Pharmacology, 667 (1-3), 410-418. doi: http://doi.org/10.1016/ j.ejphar.2011.05.025

Received date 16.07.2021

Accepted date 08.10.2021

Published date 29.10.2021

Mykhailo Myha, Postgraduate Student, Department of Pharmacognosy, National University of Pharmacy, Pushkinska str., 53, Kharkiv, Ukraine, 61002

Oleh Koshovyi*, Doctor of Pharmaceutical Sciences, Professor, Head of Department, Department of Pharmacognosy, National University of Pharmacy, Pushkinska str., 53, Kharkiv, Ukraine, 61002

Yevhen Karpun, Assistant, Department of Natural Sciences for Foreign Students and Toxicological Chemistry, Zaporizhzhia State Medical University, Maiakovskoho ave., 26, Zaporizhzhia, Ukraine, 69035

Alla Kovaleva, Doctor of Pharmaceutical Sciences, Professor, Department of Pharmacognosy, National University of Pharmacy, Pushkinska str., 53, Kharkiv, Ukraine, 61002

Olga Mala, PhD, Associate Professor, Department of Botany, National University of Pharmacy, Pushkinska str., 53, Kharkiv, Ukraine, 61002

Volodymyr Parchenko, Doctor of Pharmaceutical Sciences, Professor Department of Natural Sciences for Foreign Students and Toxicological Chemistry, Zaporizhzhia State Medical University, Maiakovskoho ave., 26, Zaporizhzhia, Ukraine, 69035

Oleksandr Panasenko, Doctor of Pharmaceutical Sciences, Head of Department, Department of Natural Sciences for Foreign Students and Toxicological Chemistry, Zaporizhzhia State Medical University Maiakovskoho ave., 26, Zaporizhzhia, Ukraine, 69035

Vera Bunyatyan, PhD, Associate Professor, I. M. Sechenov First Moscow State Medical University of the Ministry of Health of the Russian Federation, Trubetskaya str., 8, Moscow, Russian Federation, 119991

Sergiy Kovalenko, Doctor of Chemical Sciences, Professor, Department of Organic Chemistry, V. N. Karazin Kharkiv National University, Svobody sq., 4, Kharkiv, Ukraine, 61022

*Corresponding author: Oleh Koshovyi,e-mail: oleh.koshovyi@gmail.com 